\title{
Analysis of Sagittal Spinal Morphology and Function of Adolescents: A Cross-Sectional Study
}

\section{Qiang Feng ( $\nabla$ fengqiang@ciss.cn )}

China Institute of Sport Science https://orcid.org/0000-0002-0641-7080

\section{Yu Zhou}

National institute of education Science

\section{Fubaihui Wang}

China Institute of Sport Science

\section{Minghao Wang}

Tianjin university of technology

\section{Research article}

Keywords: thoracic kyphosis, adolescent, spinal morphology, spinal disorder

Posted Date: March 19th, 2020

DOI: https://doi.org/10.21203/rs.2.20879/v2

License: (9) This work is licensed under a Creative Commons Attribution 4.0 International License. Read Full License 


\section{Abstract}

Background: Spine health is significant in adolescent health. Few studies have focused on adolescent sagittal plane health; therefore, this study investigated the sagittal spine morphology and function of adolescents. Methods: This cross-sectional study analysed 1152 junior and senior high school students (543 boys and 609 girls) in Beijing. Spinal sagittal morphology and function of adolescents were measured using the Spinal Mouse. The sacral angle (SA), thoracic kyphosis angle (TKA), lumbar lordosis angle (LLA), inclination angle (INA), sacral range of motion (SROM), thoracic range of motion (TROM), lumbar range of motion (LROM), and inclination range of motion (IROM) were measured. The Matthiass strength test was used to measure changes in angles after external load placement on the adolescent spine.Results: Abnormal TKA rates were $48.2 \%$ and $44.7 \%$ in the junior and senior high school groups, respectively. Abnormal LLA rates were $44.6 \%$ and $55.4 \%$, respectively. Spinal mobility test results of the junior high school group were $60.6^{\circ} \pm 19.1^{\circ}$ (SROM), $23.0^{\circ} \pm 16.6^{\circ}$ (TROM), $81.3^{\circ} \pm 15.4^{\circ}$ (LROM), and $136.1^{\circ}$ $\pm 16.9^{\circ}$ (IROM) for boys and $66.0^{\circ} \pm 34.4^{\circ}$ (SROM), $14.0^{\circ} \pm 17.3^{\circ}$ (TROM), $66.3^{\circ} \pm 18.6^{\circ}$ (LROM), and $127.4^{\circ}$ $\pm 26.8^{\circ}$ (IROM) for girls. For the high school group, these results were $66.0^{\circ} \pm 17.6^{\circ}$ (SROM), $21.6^{\circ} \pm 16.0^{\circ}$ (TROM), $77.9^{\circ} \pm 15.1^{\circ}(\mathrm{LROM})$, and $138.4^{\circ} \pm 16.0^{\circ}$ (IROM) for boys and $72.2^{\circ} \pm 26.9^{\circ}$ (SROM), $15.7^{\circ} \pm 17.2^{\circ}$ (TROM), $65.8^{\circ} \pm 18.2^{\circ}$ (LROM), and $133.4^{\circ} \pm 21.7^{\circ}$ (IROM) for girls. There were significant differences between boys and girls $(P<0.05$ or $P<0.01)$. The Matthiass strength test results indicated that the inclination angle change (INAC) values of the junior high school group were $5.7^{\circ} \pm 5.0^{\circ}$ and $2.6^{\circ} \pm 3.7^{\circ}$ for boys and girls, respectively; however, the INAc values of the senior high school group were $2.8^{\circ} \pm 3.3$ and $1.6^{\circ} \pm 3.0$ for boys and girls, respectively, indicating significant differences $(P<0.01)$. The canonical correlation coefficients of SA, SROM, LROM, and IROM were 1.3877, -2.5384, -0.6625, and 1.6336, respectively. SROM and LROM were negatively correlated and IROM was positively correlated with spinal function.Conclusion: Thoracic kyphosis and lumber lordosis rates were high. During flexion and extension, Boys had better thoracic and lumbar vertebral activity and overall activity.

Girls had better ability to maintain the normal spinal shape with an extra load. Sacral obliquity and pelvic position greatly influence the spinal morphology.

\section{Background}

Spine health is important for both adults and adolescents. Many diseases related to the spine, such as neck pain, back pain, and scoliosis, affect the health of children and adolescents [1-4]. The spine is the backbone of the human body, and poor lifestyle habits, lack of exercise, and poor nutrition may lead to a decline in spine health, resulting in nonspecific back pain and abnormal spinal physiological curvature [5, 6]. Recent increases in sedentary lifestyles [7] have increased the prevalence of obesity [8], thereby affecting the body posture and spine health of adolescents. This study showed that the incidence of low back pain is high among Chinese adolescents and that spinal morphology (such as thoracic kyphosis) and spinal function (lumbar mobility) are correlated with nonspecific low back pain [9]. Furthermore, the sagittal morphology of the spine could negatively affect the pulmonary function among children and adolescents with certain specific diseases, such as cystic fibrosis and adolescent idiopathic scoliosis [10, 
11]. Although evidence is lacking, it could be inferred that this phenomenon may also affect healthy children and adolescents. Moreover, there is a correlation between the sagittal morphology of the spine and coronal scoliosis;for instance, sagittal spinal morphology had led to more severe scoliosis and higher rates of lumbar lordosis and thoracic kyphosis in adolescents [13]. Anomalies in the sagittal plane are likely to lead to more severe spinal diseases and changes in the morphology of the thoracic sagittal plane, which may trigger idiopathic scoliosis $[14,15]$. One previous study indicated that hypokyphosis is a risk factor of thoracic idiopathic scoliosis [16]. Another study indicated that hypokyphosis could increase the axial rotation [17]. The morphology and function of the sagittal spinal plane have important roles in adolescent spine health. However, at present, there are few quantitative studies of the sagittal morphology and function of the adolescent spine. Therefore, this study quantitatively measured the physiological curvature of the spine and its basic functions in adolescents to determine the status of adolescent spine health among Chinese students.

\section{Method}

\section{Study design}

The complete protocol of this cross-sectional study (registration no. ChiCTR1900021700) was approved by the academic board of the China Institute of Sports Science (no. 201518-21). All methods were performed in accordance with the guidelines and regulations of the institution. Before the study, written informed consent was obtained from all study participants and their parents. We excluded all students who refused to participate in the study. During the analysis, all participants were deidentified.

\section{Participants}

From September 2014 to September 2016, the middle schools in the Xicheng District of Beijing were divided into demonstration, ordinary, and general schools. Three schools at each level were selected by random sampling. At each school, three classes (junior 2, senior 1, and senior 2) were selected by simple random sampling. A cluster survey of the selected classes was conducted to test spine morphology and function.

Before the test, all students and parents were given questionnaires (the main contents of the questionnaires included questions about whether the student had a definite diagnosis of spinal diseases, whether there had been back or lumbar pain during the past month, whether there had been an acute back injury during the past month, whether there had been sports-related injuries to other body parts during the past month, and others).

\section{Sample size}

During the pre-experiment, which included 34 boys and 28 girls, the mean thoracic kyphosis angle (TKA) values were $37.3^{\circ}$ and $35.4^{\circ}$ for boys and girls, respectively. The standard deviation was 10.2 for all 
participants. With a type I error rate of 0.05 and power of $80 \%$, the study used the following formulas to compute the sample size:

$$
n_{A}=\kappa n_{B} \text { and } n_{B}=\left(1+\frac{1}{\kappa}\right)\left(\sigma \frac{z_{1-\alpha / 2}+z_{1-\beta}}{\mu_{A}-\mu_{B}}\right)^{2}
$$

As a result, each group required at least 450 participants because of the $10 \%$ rejection rate. Our design included at least 500 male and female participants.

\section{Exclusion criteria}

Participants completed screening questionnaires and were examined by physical therapists. The exclusion criteria were as follows: history of spine fracture, spine-related surgery, shoulder joint motion injury, pelvis-related injury, or definitive diagnosis of spine-related diseases (such as cauda equina syndrome, lumbar disc herniation, spinal stenosis, congenital scoliosis, idiopathic scoliosis); spine pain during the past month; acute spine-related injuries during the past month; limb fracture, joint trauma, and other phenomena during the past month; structural kyphosis with obvious structural abnormalities; positive Adams flexion test result (to exclude possible scoliosis); and anomalous kyphosis with thoracic deformity.

\section{Test methods to determine spinal morphology and function}

The gold standard of spinal morphology testing is X-ray irradiation, but the radiation produced is contrary to the ethical requirements. Therefore, in this study, a non-invasive device called the Spinal Mouse, which has good reliability and validity, was used to test the spine morphology and function [18-20]. Several previous studies have used the Spinal Mouse to measure the spinal alignment and function of different types of participants $[9,21-23]$.

Participants were asked to remove their shoes and jackets to expose the entire back from the seventh cervical vertebra (C7) to the third sacral vertebra (S3). We located the iliospinale posterius, at the same level of the S2 spinous process; then, we located the S3 down to S2 (approximately $2 \mathrm{~cm}$ below) and around the anal crease $[19,24]$. Surface localization of all spinous processes from C7 to S3 was marked with a fluorescent pen. The test protocol included the standing sagittal plane, maximum flexion, maximum extension, and Matthiass test. All body position tests determined all the spinous process markers on the body surface; then, tests were performed to assess the spine based on those markers. The specific testing methods for each body position were as follows: upright position, study participants were instructed to stand upright in a casual position with feet shoulder-width apart bearing equal weight, arms by their sides, and looking straight ahead; forward bending position, study participants were instructed to stand with their feet shoulder-width apart and to bend the torso forward while keeping the legs straight 
and allowing the arms to fall naturally; extended position, study participants were instructed to stand with their feet shoulder-width apart bearing equal weight, arms by their sides or supported by the hips, looking straight ahead, with the jaw close to the chest, and to stretch the torso backwards as far as possible; and the Matthiass test. During Matthiass test, study participants were instructed to stand with their feet shoulder-width apart, looking straight ahead. When asked, the participants pushed their arms forward horizontally while holding a certain amount of weight ( $5 \%$ of participant's body weight) until their arms created a $90^{\circ}$ angle with the shoulders. Spinal curvature and pelvic tilt were measured. Participants were instructed to retain this posture for 30 seconds; then, the second spinal curvature and pelvic tilt were measured. The Matthiass test is a useful method for detecting spinal posture changes related to strength levels $[9,25,26]$.

\section{Indexes}

The test indexes included the TKA, lumbar lordosis angle (LLA), sacral/hip angle (SA), and incline angle (INA) of the sagittal plane while the participants were in the standing and sitting positions. The TKA is the Cobb angle from $\mathrm{T} 1$ to $\mathrm{T} 12$, and the LLA is the Cobb angle from $\mathrm{L} 1$ to $\mathrm{S} 1$. The SA reflects pelvic positioning (the angle between the surface contour line and the vertical line of the sacrum) and pelvic mobility as a result of the limited movement of the sacroiliac (SI) joint. The incline line is the line between $\mathrm{T} 1$ and S1. The angle between the incline line and the vertical line is called the INA. The range of motion (ROM) of the thoracic spine and the ROM of the lumbar spine in the frontal plane were recorded. The ROM values of the sacrum, thoracic spine, and lumbar spine from the fully flexed position to the fully extended position in the sagittal plane, which can reflect the overall mobility of the spine, were also recorded. The Matthiass test was used to measure the changes in the TKA, LLA, SA, and INA upon loading.

\section{Normal ranges for the TKA and LLA}

The normal range of the TKA is $20^{\circ}-40^{\circ}[27,28]$. However, few studies have reported the normal range of the LLA for adolescents. Jean et al. reported that the mean normal LLA was $48.0^{\circ} \pm 11.7^{\circ}$ [29]; therefore, in this study, the normal range for the LLA was based on that result (mean value \pm 2 standard deviations).

\section{Statistical methods}

We created groups based on sex and grade. Because of the differences in sexes and grades, there was great diversity in the lifestyles of the Chinese students involved in this study. Using SPSS 19.0 statistical software, an independent sample t test was performed using height, weight, and age to test whether there were significant differences among the groups. An independent samples t-test was used for all characteristics of those with the same sex and age. The likelihood ratio chi-square test was used to analyse whether there were differences in the incidence of abnormal thoracic kyphosis and lumbar lordosis among the different groups. A simple correlation analysis was performed for age and spinal morphology and function. The difference was statistically significant $(P<0.05)$. 
SAS 9.4 was used for data cleaning and analysis. The CANCORR process was used to analyse four indexes (sacral INA, TKA, lumbar lordosis angle, INA) reflecting spinal morphology and eight indexes (sacral activity, thoracic activity, lumbar activity, INA activity, sacral inclination load, thoracic kyphosis load, and lumbar lordosis load) reflecting spinal function.

\section{Results}

\section{Characteristics of the study participants}

Students who met the test criteria and agreed to participate in the test underwent spinal morphology and function tests. Altogether 502 junior and 670 senior high school students were tested; 490 junior (244 boys, 246 girls) and 662 senior high school students (299 boys and 363 girls) effectively completed the testing. Twenty participants did not complete the testing and were excluded from the analysis.

The basic information of the participants is shown in Table 1. There were no significant differences in height, weight, or body mass index (BMI) between the groups.

\section{Sagittal plane morphology and abnormal rates}

The results of the spine morphology test in the upright position are shown in Table 2. In the junior high school group, the INA of girls was smaller and significantly different than that of boys $(P<0.01)$. The INA of the high school group was smaller and significantly different than that of the junior high school group ( $P<0.05$ for boys; $P<0.01$ for girls).

Abnormal thoracic kyphosis and lumbar lordosis rates are shown in Table 3. In the high school group, the abnormal TKA rate of girls was lower than that of boys. Additionally, among the girls, the abnormal TKA rate of the high school group was lower than that of the junior high school group $(P<0.01)$.

\section{Spine function (strength and range of motion)}

The spinal function of adolescents was evaluated using the spine mobility test and Matthiass strength test. The results of the spine mobility test are shown in Table 4. The sacral mobility of the boys was less than that of the girls, whereas the ranges of thoracic angles, lumbar angles, and INA of boys were larger than those of the girls, and the difference was significant $(P<0.05$ or $P<0.01)$. The sacral mobility of the high school group was greater than that of the middle school group, whereas the lumbar mobility of the high school group was less than that of the middle school group, and the difference was significant $(P<0.01)$. Among the girls, the sacral mobility and INA of the high school group were greater than those of the middle school group, and the difference was significant $(P<0.05$ or $P<0.01)$.

The Matthiass test results are shown in Table 5. Changes in the sacral INA, TKA, and INA of the girls in the junior high school group were smaller than those of the boys (significantly different; $P<0.01$ ). In the senior high school group, only the changes in the sacral INA were smaller in the girls than in the boys (significantly different; $\mathrm{P}<0.01$ ). The spinal motion change of the high school group was smaller than that 
of the middle school group among boys. The INA of the high school group was smaller than that of the middle school group among girls.

\section{Canonical correlation analysis of spinal sagittal morphology and function}

The likelihood ratio method involving four sets of typical variables was used to test whether the difference between the canonical correlation coefficient and zero was significant (Table 6). The first typical variable provides $62.6 \%$ of the relevant information. The variation explained by the first set of typical variables accounts for $62.6 \%$ of the total variation (variance), indicating a dominant role. Therefore, the first set of typical variables was further analysed. The specific results are shown in Tables 7 and 8.

As shown in Tables 7 and 8 , the first typical variable (Form1) of the spinal morphological index was Form $1=1.3877 *$ sacral inclination $*+0.4332 *$ thoracic kyphosis $*+0.4683 *$ lumbar

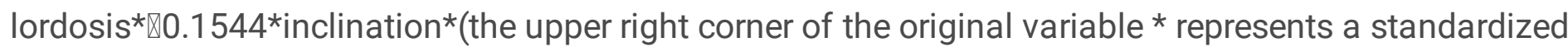
variable). The results showed that in Form1, the typical coefficient of sacral inclination was larger, indicating that it had a larger role in the first typical variable. Form 1 mainly represented the sacral inclination index, and its value positively correlated with spinal morphology.

The first typical variable of the spine function index (Function 1 ) is Function $1=\varangle 2.5384^{\star}$ sacral

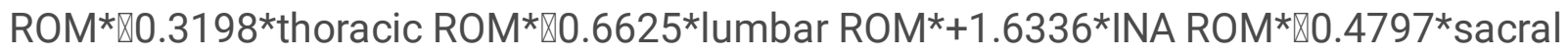

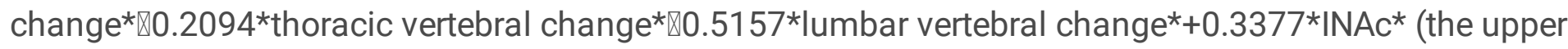
right corner * of the original variable represents the standardized variable). The results showed that the typical coefficients of sacral ROM, INA ROM, and lumbar ROM in Function1 were larger, which indicated that Function 1 had a larger role. Function 1 mainly represents sacral ROM, INA ROM, lumbar ROM, and other indicators. However, because the coefficients of sacral ROM and lumbar ROM were negative, they negatively correlated with spinal function, whereas INA ROM positively correlated with spinal function.

The canonical correlation coefficient of Form 1 and Function 1 is $0.442(\mathrm{P}<0.0001)$, which indicates a positive correlation between the sacral INA of spinal morphology and the ROM of spinal function, but a negative correlation with sacral ROM and lumbar ROM.

\section{Discussion}

At present, there are only a few studies on the morphology of the sagittal spine in adolescents, and there are no corresponding quantitative screening criteria for curvature abnormalities of the sagittal spine. This study showed that the rates of abnormal thoracic kyphosis and lumbar lordosis are high ( $>45 \%)$, except for girls in the junior high school group. The LLA values of the junior high school group were $22.8 \pm 8.1$ and $23.5 \pm 9.9$ for boys and girls, respectively; however, those of the senior high school group were $22.9 \pm 7.8$ and $23.7 \pm 11.2$, respectively. There was a certain gap between these values and the standard values reported by foreign scholars. Although this study did not use X-rays for testing, the test method 
used has sufficient reliability and validity and can reflect the status of adolescent spinal morphology in the sagittal plane.

The high incidence of sagittal morphological abnormalities may be related to the prevalence of sedentary behaviour and screen time. Long sitting times are likely to have adverse effects on the spinal development of adolescents, who are still growing. If the correct sitting posture is not maintained, then the normal S-shape of the spine may be altered, thereby affecting the normal biomechanical structure of the human spine. One study showed that the formation of excessive kyphosis of the thoracic spine is closely related to poor daily posture [30]. Another study assessed the differences in the spine shape in a sitting posture and a standing posture during the growth and development of children and adolescents and reported significant differences in some segments of the spine in the sagittal plane [31]. The adolescent skeleton is susceptible to bad posture, which may lead to excessive kyphosis of the thoracic spine and decreased lumbar curvature. Abnormalities of the TKA comprise a common phenomenon. Additionally, obesity could be a risk factor for abnormalities. Valdovino et al suggested that increased $\mathrm{BMI}$ is associated with increased thoracic kyphosis in adolescent scoliosis patients and adolescents without scoliosis [32]. Considering the fast growing obesity prevalence in China [33], there could be some correlation with abnormalities of the spine. We could not determine the exact reasons for the high thoracic kyphosis and lumbar lordosis rates; therefore, more studies should be performed in future,

Muscle strength is closely related to human health, and strength has an impact on adolescent spine health. Studies have shown that better back muscle endurance can protect against back pain [34]. In this study, the Matthiass strength test results showed that the girls were better at maintaining the correct shape of the spine against external force. This result may indicate that the girls had stronger small muscle groups than the boys. According to China's physical education syllabus and fitness monitoring of students, the main emphasis is on the maximum strength and explosive power of large muscle groups; although sit-ups and standing long jump exercises use the core muscles, the deep small muscle group is not sufficiently activated or effectively trained. Effective exercise methods for maintaining the strength and quality of the small muscle groups that contribute to the correct shape of the spine are lacking. Several studies have shown that the small muscle groups, especially the stable muscle groups in the core area of the body, are closely related to spine health [35-37]. This study showed poor small muscle strength among the participants, which may suggest that the coordinated development of the small and large muscles should be emphasized in future physical education reforms and adolescent health interventions.

This study explored the relationship between spinal morphology and function in adolescents through a canonical correlation analysis. Most importantly, sacral inclination positively correlated with spinal morphology; that is, within a certain range, the larger the sacral inclination, the better the spinal morphology. Sacral obliquity is the angle between the upper end plate and the horizontal line of S1 [38, 39], and studies have shown that it may be associated with low back pain [40]. Furthermore, the Spinal Mouse test indicated that sacral inclination was highly correlated with the pelvic position, and that sacral inclination was more often present than absent when the pelvis was excessively retroverted. It can be 
inferred from the experimental results that there may be a small sacral inclination (i.e., pelvic retroversion) in adolescent spines. The phenomenon of pelvic retroversion may also affect the curvature of the lumbar spine in adolescents, thus affecting the shape of the entire spine. Sacral obliquity is seldom involved in the study of the sagittal and stiff surface morphology of the spine. However, Barrey et al. found that a decrease in sacral obliquity and increase in pelvic obliquity in adolescents were significantly different from those of normal adults [41]. Moreover, sacral obliquity is highly correlated with lumbar lordosis [42]. Whether sacral inclination affects the shape and function of lumbar lordosis in adolescents deserves further study. Based on the results of this study, spine health intervention efforts should focus on sacral inclination and pelvic position to correct the abnormal spinal morphology caused by the sacrum and pelvis.

The first canonical correlation of spinal function indicated that the greater the sacral mobility and lumbar mobility, the worse the spinal function. The stability of the lumbar spine should be maintained as much as possible during sports because it is closely related to the function of the spine and the prevention of back pain [43]. The results of this study are consistent with this idea. As the spine progresses from full flexion to full extension, the sacrum and lumbar spine should remain as stable as possible; the greater the activity, the worse the spinal function. In this study, the degree of tilt activity represented the change in the tilt line and reflected the overall degree of activity. Because the cervical spine, lumbar spine, and lumbosacral joints compensate for one another (in part), the change in tilt angle cannot be simply understood as the sum of all joint activities; rather, it reflects the activity of the thoracic, lumbar, and lumbosacral joints (sacrum/hip joint), as the spine moves from flexion to extension. Because it is assumed that sacral mobility is negatively correlated with lumbar mobility and spinal function, there may be a lack of thoracic mobility in adolescents. According to the Selective Functional Movements Assessment (SFMA) system, spinal motion requires the participation of all segments; the absence of function of any segment will inevitably impact the overall spinal function[44-46]. For example, the results of this study suggested that adolescents who perform flexion-extension exercises use excessive lumbar spine compensation due to inadequate activity of the thoracic spine, thus affecting the overall function of the spine. Recently, insufficient activity of the thoracic vertebrae has attracted the attention of scholars. Studies have shown that increased activity of the thoracic spine has an important role in improving spinerelated problems, such as nonspecific low back pain and neck pain[44, 47]. Therefore, insufficient thoracic spine activity is likely to be prevalent among adolescents. Future spinal-related interventions should consider thoracic spine mobility to achieve better prevention and treatment.

The canonical correlation analysis of spine morphology and function also showed that, within a certain range, the greater the sacral INA of spine morphology, the greater the degree of motion of the INA. This suggests that sacral inclination is a factor that restricts the overall ROM of the spine in adolescents. In this study, sacral obliquity can be considered somewhat related to pelvic position [39, 48]; the smaller the sacral obliquity, the smaller the pelvic retroversion. Whether long-term sedentary behaviour or other unhealthy lifestyle factors of adolescents result in pelvic retroversion that restricts the overall ROM of the spine during movement deserves further consideration and research. Each segment of the spine interacts with the others during daily activities [49], and the results of this study support this idea. Mobility with 
lumbar lordosis and sacral inclination (which represents pelvic mobility) are mutually constrained. The mobility of the pelvis increases but the mobility of the lumbar spine decreases, and vice versa. The lumbar spine should be stable, whereas the pelvis and hip joints should be more flexible $[49,50]$. If the pelvic mobility of adolescents is limited, then the adjacent lumbar spine is likely to compensate for the increased mobility to maintain motor ability. This adaptive change may lead to nonspecific low back pain and other spine-related diseases $[43,45]$. Therefore, adolescent spine health intervention efforts should improve pelvic mobility during exercise to reduce the pressure of the lumbar spine.

When the data of the junior and senior high school groups were compared in terms of spinal physiological curvature, the INA index of the boys and that of the girls in the senior high school group were significantly smaller than those of boys and girls in the junior high school group. The tilt angle represents the degree of forward and backward tilt of the body's centre of gravity. The smaller the tilt angle, the greater the range of backward tilt of the body's centre of gravity. With increasing age, adolescents have a tendency toward retroverted centres of gravity, which may be related to their abnormal body posture. For example, the long periods spent in the supine position may cause upper and lower crossing syndrome, which may affect the body's centre of gravity $[5,6,51]$. At present, there are a few studies on the standard value of the correct body posture and tilt angle for the whole population. It is important to determine the standard angle of the adolescent centre of gravity to promote spine health. In terms of the strength index, the high school group was better than the junior high school group at maintaining the basic form of the spine; this was especially true for the boys. The degree of spinal deformation of each spinal segment in 30 seconds was lower in the high school group than in the junior high school group. Although the female students had lower scores, the difference was not statistically significant. The results suggested that with increasing age, the boys' ability to maintain the physiological curvature of the spine improved, whereas the ability of the girls remained the same with age. It has been reported that increasing age is a risk factor for low back pain [4]. Furthermore, some studies have shown different rates of spinal pain based on age among girl [52-54]. The results of this study, which indicated that the strength of the small muscle group did not improve with age and was related to the higher prevalence spinal pain, need further consideration.

There were some limitations to this study. Although the reliability and validity of the Spinal Mouse test have been recognized, there is a notable difference between this method and the gold standard (X-ray examination). It is important to find a more reliable evaluation method that can effectively replace the use of X-rays for the future study of adolescent spinal morphology and function. Furthermore, this study included middle school students in the Xicheng District of Beijing. The Xicheng District comprises residents with a higher educational level compared to other areas of China, and it is a leader in terms of quality education promotion. In other areas of China, students face greater learning pressure and schoolwork burden. Whether the sagittal shape and function of their spines will be affected is uncertain. Therefore, more scientific research should be performed to identify the morphological and functional characteristics of the sagittal spine of Chinese students to obtain reliable data to promote adolescent spine health. 


\section{Conclusion}

Compared with the standards for thoracic kyphosis and lumbar lordosis, the rates of abnormal thoracic kyphosis and lordosis in the adolescents who participated in our study are high. Moreover, during the weight-bearing test, girls were better than boys at maintaining the strength of stable muscle groups with normal spine morphology with an extra load. During the motion from flexion to extension, the ROM of the thoracic spine and lumbar spine and the total ROM were better in boys than in girls, and the decrease in ROM of the thoracic spine may have a greater impact on the function of the adolescent spine. In the upright posture, girls in junior and senior high schools showed a tendency toward a more forward centre of gravity. The SA, which reflects the pelvic position, had a great impact on adolescent spinal morphology. Our data provide a reference value for the development of postural spine health screening among adolescents and provide a direction for preventing serious adolescent spine-related diseases and reducing the risk of spine-related problems in adulthood.

\section{List Of Abbreviations}

sacral angle SA

thoracic kyphosis angle TKA

lumbar lordosis angle LLA

inclination angle INA

sacral range of motion SROM

thoracic range of motion TROM

lumbar range of motion LROM

inclination range of motion IROM

sacral angle change Sac

thoracic angle change TKAc

lumbar lordosis angle change LLAc

inclination angle change INAC

\section{Declarations}

\section{Ethics approval and consent to participate}


The complete protocol of this cross-sectional study was approved by the academic board of the China Institute of Sports Science (no. 201818-21). The written informed consent indicating the purpose and methods of this study was provided to students and parents. Participants who signed the consent were included in our study.

\section{Consent for publication}

The data of this study do not contain any personal information.

\section{Availability of data and materials}

The datasets analysed during the current study are available from the corresponding author on reasonable request.

\section{Competing interests}

The authors declare that they have no competing interests.

\section{Funding}

The study was funded by China Institute of Sport Science (19-14,19-21). The funding institute was not involved in the design of the study, collection of data, analysis of data, interpretation of data, or writing of the manuscript.

\section{Authors' contributions}

QF designed the whole study. QF and YZ analysed and interpreted the patient data. FW and MW contributed to writing and revising the manuscript. All authors read and approved the final manuscript.

\section{Acknowledgements}

The authors are thankful for the funding from the China Institute of Sport Science. The authors thank all the individuals who participated in this study.

\section{References}

1. Yilmaz, H., et al., Prevalence of adolescent idiopathic scoliosis in Turkey: an epidemiological study. Spine J, 2020.

2. Zheng, Y., et al., Epidemiological study of adolescent idiopathic scoliosis in Eastern China. Journal of rehabilitation medicine, 2017. 49(6): p. 512-519.

3. Gheysvandi, E., et al., Neck and shoulder pain among elementary school students: prevalence and its risk factors. BMC Public Health, 2019. 19(1): p. 1299.

4. Ben Ayed, H., et al., Prevalence, Risk Factors and Outcomes of Neck, Shoulders and Low-Back Pain in Secondary-School Children. J Res Health Sci, 2019. 19(1): p. e00440. 
5. Ruivo, R.M., P. Pezarat-Correia, and A.I. Carita, Cervical and shoulder postural assessment of adolescents between 15 and 17 years old and association with upper quadrant pain. Brazilian journal of physical therapy, 2014. 18(4): p. 364-371.

6. Odebiyi, D.O., O.A. Olawale, and Y.M. Adeniji, Impact of Computer Related Posture on the Occurrence of Musculoskeletal Discomfort among Secondary School Students in Lagos, Nigeria. Nig Q J Hosp Med, 2013. 23(4): p. 237-42.

7. Al-Thani, M., et al., Prevalence of physical activity and sedentary-related behaviors among adolescents: data from the Qatar National School Survey. Public Health, 2018. 160: p. 150-155.

8. Ponta, M.L., et al., Effects of obesity on posture and walking: study prior to and following surgically induced weight loss. Obes Surg, 2014. 24(11): p. 1915-20.

9. Feng, Q., et al., Relationship between spinal morphology and function and adolescent non-specific back pain: A cross-sectional study. J Back Musculoskelet Rehabil, 2017. 30(3): p. 625-633.

10. Barker, N., et al., Thoracic Kyphosis is Now Uncommon Amongst Children and Adolescents with Cystic Fibrosis. Front Pediatr, 2014. 2: p. 11.

11. Xue, X., et al., An analysis of thoracic cage deformities and pulmonary function tests in congenital scoliosis. Eur Spine J, 2015. 24(7): p. 1415-21.

12. Hong, J.Y., et al., Effect of Coronal Scoliotic Curvature on Sagittal Spinal shape - Analysis of Parameters in Mature Adolescent Scoliosis Patients. 2017.

13. Hong, J.Y., et al., Effect of Coronal Scoliotic Curvature on Sagittal Spinal Shape: Analysis of Parameters in Mature Adolescent Scoliosis Patients. Clin Spine Surg, 2017. 30(4): p. E418-e422.

14. Deacon, P., B.M. Flood, and R.A. Dickson, Idiopathic scoliosis in three dimensions. A radiographic and morphometric analysis. J Bone Joint Surg Br, 1984. 66(4): p. 509-12.

15. Burwell, R.G., Aetiology of idiopathic scoliosis: current concepts. Pediatr Rehabil, 2003. 6(3-4): p. 13770.

16. STOKES. 1998; Available from: www.ndos.ox.ac.uk/pzs/Group_2/Conclusion.html

17. Grivas, T.B., et al., The Double Rib Contour Sign (DRCS) in lateral spinal radiographs: aetiologic implications for scoliosis. Stud Health Technol Inform, 2002. 88: p. 38-43.

18. Mizukami, S., et al., Accuracy of spinal curvature assessed by a computer-assisted device and anthropometric indicators in discriminating vertebral fractures among individuals with back pain. Osteoporos Int, 2014. 25(6): p. 1727-34.

19. Mannion, A.F., et al., A new skin-surface device for measuring the curvature and global and segmental ranges of motion of the spine: reliability of measurements and comparison with data reviewed from the literature. 2004. 13(2): p. 122-136.

20. Post, R.B. and V.J. Leferink, Spinal mobility: sagittal range of motion measured with the SpinalMouse, a new non-invasive device. Arch Orthop Trauma Surg, 2004. 124(3): p. 187-92.

21. Feng, Q., et al., The effect of a corrective functional exercise program on postural thoracic kyphosis in teenagers: a randomized controlled trial. Clin Rehabil, 2018. 32(1): p. 48-56. 
22. Demarteau, J., et al., Trunk inclination and hip extension mobility, but not thoracic kyphosis angle, are related to 3D-accelerometry based gait alterations and increased fall-risk in older persons. Gait Posture, 2019. 72: p. 89-95.

23. Yanagisawa, S., et al., Relation among the knee, sagittal spinal alignment, and the spinal range of motion: Investigation in local medical check-ups using the SpinalMouse. Asia Pac J Sports Med Arthrosc Rehabil Technol, 2015. 2(2): p. 68-71.

24. Tixa, S., Atlas D'anatomie Palpatoire T.1; Cou, Tronc, Membre Supérieur (3e Edition).

25. Albertsen, I.M., et al., Spinal posture changes using dynamic rasterstereography during the modified Matthiass test discriminate between postural weak and strong healthy children (10-14 years): a pilot study. Eur J Pediatr, 2018. 177(9): p. 1327-1334.

26. Albertsen, I.M., et al., Spinal postural changes during the modified Matthiass test in healthy children : Interday and interrater reliability of dynamic rasterstereographic measurements. Orthopade, 2018. 47(7): p. 567-573.

27. Ali, R.M., D.W. Green, and T.C. Patel, Scheuermann's kyphosis. Curr Opin Pediatr, 1999. 11(1): p. 70-5.

28. Tribus, C.B., Scheuermann's kyphosis in adolescents and adults: diagnosis and management. J Am Acad Orthop Surg, 1998. 6(1): p. 36-43.

29. Mac-Thiong, J.M., et al., Sagittal spinopelvic balance in normal children and adolescents. Eur Spine J, 2007. 16(2): p. 227-34.

30. Kamali, F., et al., Comparison of manual therapy and exercise therapy for postural hyperkyphosis: $A$ randomized clinical trial. Physiother Theory Pract, 2016. 32(2): p. 92-7.

31. Kamaci, S., et al., The Evolution of Sagittal Spinal Alignment in Sitting Position During Childhood. Spine (Phila Pa 1976), 2015. 40(13): p. E787-93.

32. Valdovino, A.G., et al., Obesity Is Associated With Increased Thoracic Kyphosis in Adolescent Idiopathic Scoliosis Patients and Nonscoliotic Adolescents. Spine Deform, 2019. 7(6): p. 865-869.

33. Dong, Y., et al., Economic development and the nutritional status of Chinese school-aged children and adolescents from 1995 to 2014: an analysis of five successive national surveys. Lancet Diabetes Endocrinol, 2019. 7(4): p. 288-299.

34. Lardon, A., C. Leboeuf-Yde, and C. Le Scanff, Is back pain during childhood or adolescence associated with muscle strength, muscle endurance or aerobic capacity: three systematic literature reviews with one meta-analysis. Chiropr Man Therap, 2015. 23: p. 21.

35. Gur, G., C. Ayhan, and Y. Yakut, The effectiveness of core stabilization exercise in adolescent idiopathic scoliosis: A randomized controlled trial. Prosthet Orthot Int, 2017. 41(3): p. 303-310.

36. Majeed, A.S., et al., The effectiveness of a simplified core stabilization program (TRICCS-Trivandrum Community-based Core Stabilisation) for community-based intervention in chronic non-specific low back pain. J Orthop Surg Res, 2019. 14(1): p. 86.

37. Schmid, A.B., et al., Paraspinal muscle activity during symmetrical and asymmetrical weight training in idiopathic scoliosis. J Sport Rehabil, 2010. 19(3): p. 315-27. 
38. Kim, H.J., et al., Influences of trunk muscles on lumbar lordosis and sacral angle. Eur Spine J, 2006. 15(4): p. 409-14.

39. Gilliam, J., et al., Relationship of the pelvic angle to the sacral angle: measurement of clinical reliability and validity. J Orthop Sports Phys Ther, 1994. 20(4): p. 193-9.

40. Azar, M., et al., Association of low back pain with lumbar lordosis and lumbosacral angle. Journal of Mazandaran University of Medical Sciences, 2010. 20: p. 9-15.

41. Barrey, C., et al., Sagittal balance of the pelvis-spine complex and lumbar degenerative diseases. $A$ comparative study about 85 cases. Eur Spine J, 2007. 16(9): p. 1459-67.

42. Bron, J.L., B.J. van Royen, and P.I. Wuisman, The clinical significance of lumbosacral transitional anomalies. Acta Orthop Belg, 2007. 73(6): p. 687-95.

43. Page, P., C. Frank, and R. Lardner, Assessment and treatment of muscle imbalance: The Janda approach. Champaign: Human Kinetics, 2009: p. 27-30.

44. Lee, K.W. and W.H. Kim, Effect of thoracic manipulation and deep craniocervical flexor training on pain, mobility, strength, and disability of the neck of patients with chronic nonspecific neck pain: a randomized clinical trial. J Phys Ther Sci, 2016. 28(1): p. 175-80.

45. Coglianese, D., Rehabilitation of the Spine: A Practitioner's Manual, ed 2. Physical Therapy, 2007. 87 : p. 479-479.

46. Goshtigian, G.R. and B.T. Swanson, USING THE SELECTIVE FUNCTIONAL MOVEMENT ASSESSMENT AND REGIONAL INTERDEPENDENCE THEORY TO GUIDE TREATMENT OF AN ATHLETE WITH BACK PAIN: A CASE REPORT. Int J Sports Phys Ther, 2016. 11(4): p. 575-95.

47. Mohanty, P.P. and M. Pattnaik, Mobilisation of the thoracic spine in the management of spondylolisthesis. J Bodyw Mov Ther, 2016. 20(3): p. 598-603.

48. Zawojska, K., et al., Correlation of Body Mass Index with Pelvis and Lumbar Spine Alignment in Sagittal Plane in Hemophilia Patients. Medicina (Kaunas), 2019. 55(10).

49. Izraelski, J., Movement: Functional Movement Systems: Screening, Assessment, and Corrective Strategies. The Journal of the Canadian Chiropractic Association, 2012. 56: p. 158-158.

50. Myers, T., The 'anatomy trains'. Journal of Bodywork and Movement Therapies, 2002. 1: p. 91-101.

51. Ruivo, R.M., P. Pezarat-Correia, and A.I. Carita, Effects of a Resistance and Stretching Training Program on Forward Head and Protracted Shoulder Posture in Adolescents. J Manipulative Physiol Ther, 2017. 40(1): p. 1-10.

52. Trevelyan, F.C. and S.J. Legg, The prevalence and characteristics of back pain among school children in New Zealand. Ergonomics, 2010. 53(12): p. 1455-60.

53. Gustafsson, M.L., et al., The prevalence of neck-shoulder pain, back pain and psychological symptoms in association with daytime sleepiness - a prospective follow-up study of school children aged 10 to 15. Scand J Pain, 2018. 18(3): p. 389-397.

54. Stallknecht, S.E., et al., Spinal pain and co-occurrence with stress and general well-being among young adolescents: a study within the Danish National Birth Cohort. Eur J Pediatr, 2017. 176(6): p. 
807-814.

\section{Supplementary Files}

This is a list of supplementary files associated with this preprint. Click to download.

- checklist.doc

- supplementarymaterial.pdf 